\title{
RX J1643.7+3402: A new bright cataclysmic variable*
}

\author{
A. M. Mickaelian ${ }^{1}$, S. K. Balayan ${ }^{1}$, S. A. Ilovaisky ${ }^{2}$, C. Chevalier $^{2}$, M.-P. Véron-Cetty ${ }^{2}$, and P. Véron ${ }^{2}$ \\ 1 Byurakan Astrophysical Observatory and Isaac Newton Institute of Chile, Armenian branch, \\ Byurakan 378433, Armenia \\ e-mail: aregmick, sbalayan@bao.sci.am \\ 2 Observatoire de Haute-Provence, CNRS, 04870 Saint-Michel l'Observatoire, France \\ e-mail: chevalier, mira, veron@obs-hp.fr
}

Received 17 July 2001 / Accepted 30 August 2001

\begin{abstract}
We report the discovery of a new bright $(V \sim 12.6)$ cataclysmic variable star identified with the ROSAT X-ray source RX J1643.7+3402. Spectroscopic and photometric observations show it to be a novalike variable sharing some of the characteristics of the SW Sex sub-class of novalike CVs. The spectroscopic period may be either 2.575 or 2.885 , within the period "gap". A photometric modulation with a probable period of 2.595 and an amplitude of $\sim 0.1 \mathrm{mag}$ in $V$ is present on most nights and could be either a "positive" or a "negative" superhump modulation (depending on the exact spectroscopic period), indicating the presence of a precessing accretion disk in this system. Rapid variations of 0.1 to 0.2 mag amplitude in $V$ repeat with a time scale of $\sim 15 \mathrm{~min}$.
\end{abstract}

Key words. accretion, accretion disks - stars: novae, cataclysmic variables - stars: binaries: close

\section{Introduction}

In order to study the surface density of bright QSOs (Mickaelian et al. 1999), we have conducted a search for such objects in part of the area $\left(+33^{\circ}<\delta<+45^{\circ}\right)$ of the First Byurakan Survey (FBS) which was aimed at the detection of blue stellar objects (Abrahamian \& Mickaelian 1996, and references therein). To check the completeness of this sample we cross-correlated the sources in the ROSAT RASS X-ray survey (Voges et al. 1999) with the objects in the USNO-A2.0 catalog (Monet et al. 1996) brighter than $\mathrm{O}(\mathrm{USNO})=16.0$ and found a few bright additional QSO candidates. One of them, near RX J1643.7+3402, is a bright starlike object at a galactic latitude of $+40^{\circ} 2$, located 82" to the west of the bright F2 star HD 151087 (see Fig. 1). Its J2000 coordinates, measured on the Digitized Sky Survey, are: $\alpha=16^{\mathrm{h}} 43^{\mathrm{m}} 45^{\mathrm{s}} .71$ and $\delta=34^{\circ} 02^{\prime} 39^{\prime \prime} 9$; it is located $77^{\prime \prime} 2$ from the X-ray position. This identification was first proposed by Bade et al. (1998), but since the digitized spectrum from the Hamburg objective-prism survey plate was saturated no information on the nature of the object was published, and later also by Rutledge et al. (2000) who used a statistical cross-identification

Send offprint requests to: S. A. Ilovaisky, e-mail: ilovaisky@obs-hp.fr

* Partly based on observations obtained with the Byurakan Observatory 2.6-m telescope and with the OHP 1.93-m and 1.2-m telescopes. technique. The observations reported in this paper show it to be a cataclysmic variable.

\section{Observations}

\subsection{Spectroscopic observations}

On May 23, 2000, three spectra (10 min exposure each) were obtained with the ByuFOSC-2 focal reducer (Movsessian et al. 2000) attached to the prime focus of the Byurakan Observatory 2.6-m telescope; the "green" grism (4200-6900 $\AA$ ) was used giving a dispersion of $2.7 \AA \mathrm{pix}^{-1}$. On May 25, another spectrum (15 min exposure) was obtained with the "red" grism (5400-7600 ̊) giving a dispersion of $2.1 \AA \mathrm{pix}^{-1}$. The detector was a $1060 \times 514$, $24 \times 24 \mu \mathrm{m}$ pixel Thomson CCD. The spectra were flux calibrated using the standard star $\mathrm{BD}+28^{\circ} 4211$ (Stone 1977). The continuum is very blue; several emission lines are present, the strongest being $\mathrm{H} \alpha, \mathrm{H} \beta$, He II $\lambda 4686$, He I $\lambda 6678$ and the "Bowen blend" emission feature at $\sim 4645 \AA$.

During the period 22-28 September 2000 more detailed spectroscopic observations were carried out with the CARELEC spectrograph (Lemaitre et al. 1989) attached to the Cassegrain focus of the OHP 1.93-m telescope. We used a $600 \mathrm{lmm}^{-1}$ grating resulting in a dispersion of $66 \AA \mathrm{mm}^{-1}$. The detector was a $1024 \times 2048$, $13.5 \times 13.5 \mu \mathrm{m}$ pixel EEV 42-20 CCD. Exposure times 


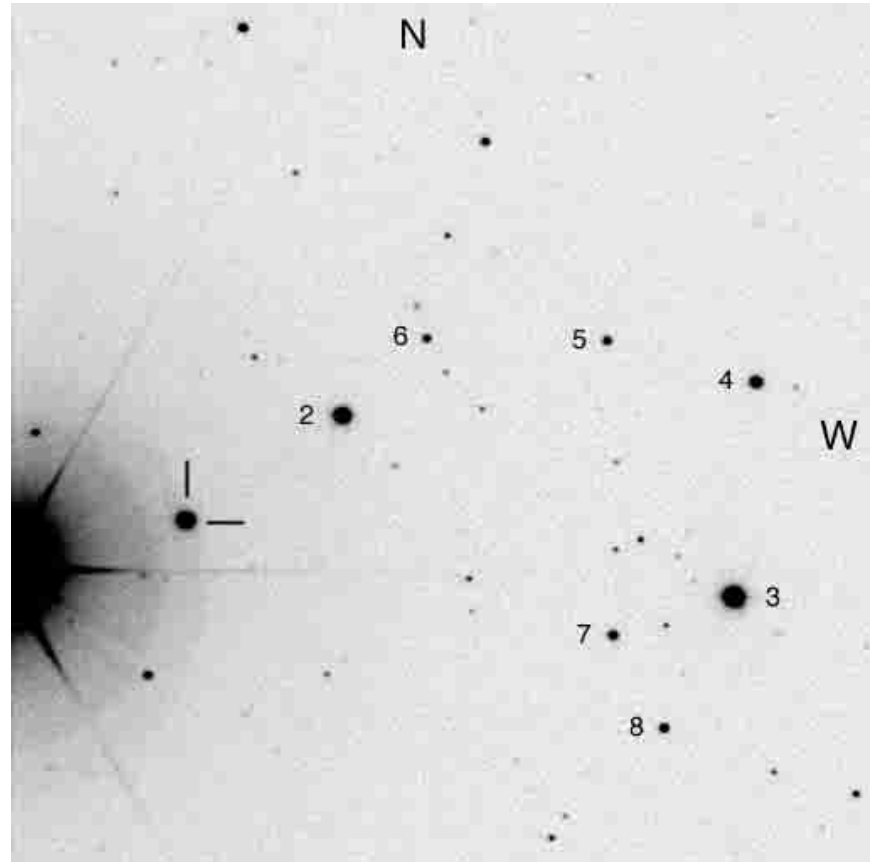

Fig. 1. Finding chart for RX J1643.7+3402. This is a $30-\mathrm{s} R_{\mathrm{c}}$ exposure taken on September 23, 2000 with the OHP $1.2-\mathrm{m}$ telescope. The field of view is $6 \times 6$ arcmin. The variable is indicated by two tick marks. Magnitudes and colors for numbered field stars are given in Table 2. Stars 2 and 3 were used as comparisons. The bright star $(V=6)$ at left is HD 151087.

were generally $10 \mathrm{~min}$. Seven columns of the CCD $\left(\sim 3^{\prime \prime}\right)$ were extracted. The slit width was 2 "' 1 , corresponding to a projected slit width on the detector of $52 \mu \mathrm{m}$ i.e. 3.8 pixels. The resolution, as measured on the night sky lines, was $\sim 3.4 \AA F W H M$. The wavelength range was 4280-6100 A. The spectra were wavelength calibrated using an Ar lamp and flux calibrated using the standard stars Feige $15, \mathrm{BD}+28^{\circ} 4211$ and EG 247, taken from Oke (1974) and Stone (1977). To obtain accurate velocity measurements, we applied wavelength corrections by measuring the $[\mathrm{O} \mathrm{I}] \lambda 5577$ night sky line on each spectrum (the corrections were in the range $-1.3,-0.4 \AA$ ).

The profiles of all emission lines are complex and variable. Some $\mathrm{H} \beta$ profiles have a flat top, others display a shoulder while a few show a clear central absorption; we derived radial velocities using our own software, as described in Gonçalves et al. (1999), by fitting each profile with a main emission component and a minor unresolved Gaussian absorption (see Sect. 5). This procedure derives velocity information essentially from the line flanks. The radial velocities for the main emission component vary between -205 and $+125 \mathrm{~km} \mathrm{~s}^{-1}$.

We coadded all our spectra (see Fig. 2). The resulting spectrum shows, in addition to the lines seen on the low resolution BAO spectra, several He I lines (4388, 4471, $4713,4921,5016$ and $5875 \AA)$ and an additional He II line $(5411 \AA)$; there is also a weak, broad ( $\sim 33 \AA F W H M)$ C IV $\lambda 5804$ emission line (this is in fact a doublet at 5801.5 and $5812.1 \AA$ ). Table 1 gives the equivalent widths $(E W)$
Table 1. Equivalent widths of the main emission lines as measured on the integrated spectrum.

\begin{tabular}{ll}
\hline Line & $E W(\AA)$ \\
\hline $\mathrm{H} \gamma$ & 1.6 \\
Bowen Blend & 2.0 \\
He II $\lambda 4686$ & 2.5 \\
He I $\lambda 4713$ & 0.12 \\
H $\beta$ & 2.8 \\
He I $\lambda 4921$ & 0.33 \\
He I $\lambda 5016$ & 0.32 \\
C IV $\lambda 5804$ & 0.62 \\
\hline
\end{tabular}

of the main emission lines measured on this spectrum. Blends of atmospheric water vapor absorption lines are present between 5680 and $5800 \AA$ and between 5870 and $6000 \AA$.

\subsection{Photometric observations}

We obtained photometric observations of the RX J1643.7+3402 field on nine nights (18-27 September 2000) using the OHP $1.2-\mathrm{m}$ telescope and a CCD TK1024 $\times 1024$ camera. The pixel size is $24 \mu \mathrm{m}$ which projects to $0^{\prime \prime} 7$ on the sky. Most of the frames used for monitoring the brightness were $30 \mathrm{~s} V$-band exposures. The effective time resolution (exposure time + read-out time) was $113 \mathrm{~s}$ on the first four nights, but on subsequent nights we used a single $512 \times 512$ detector quadrant $(6 \times 6$ arcmin field of view), which resulted in a read-out time of $30 \mathrm{~s}$ and an effective time resolution of $60 \mathrm{~s}$. The longest runs possible at this time of year were about 2.5 to $3 \mathrm{~h}$ long. Total or partial simultaneous coverage with the spectroscopic observations at the 1.93-m was achieved on six nights (22-27 September). Dome flat-fields were obtained using controlled daylight illumination. All necessary data reduction was done using the ESO-MIDAS software package.

We derived differential magnitudes for the variable star and two nearby comparison stars using custom optimalaperture photometry software. Sky values were derived for each star from concentric annuli designed to correct essentially for scattered light from the nearby bright star HD 151087. Results for our photometry runs are shown in Fig. 3 where the magnitudes, derived from $655 \mathrm{im}-$ ages, have been transformed into the standard $V$-band system. These light curves show that the object varied between $V$ magnitude 12.5 and 12.7 during our September 2000 observing run. Superimposed on a $\sim 0.1 \mathrm{mag}$ slow modulation, there are rapid variations of 0.1 to 0.2 magnitude amplitude repeating with a time scale of $\sim 15 \mathrm{~min}$.

Observations using the entire $U B V R_{\mathrm{c}} I_{\mathrm{c}}$ system filter set were secured on September 23, together with frames of standards in M 13 (Forbes \& Dawson 1986; Arp \& Johnson 1955) for photometric calibration. Table 2 gives magnitudes and colors for several stars in the field including 


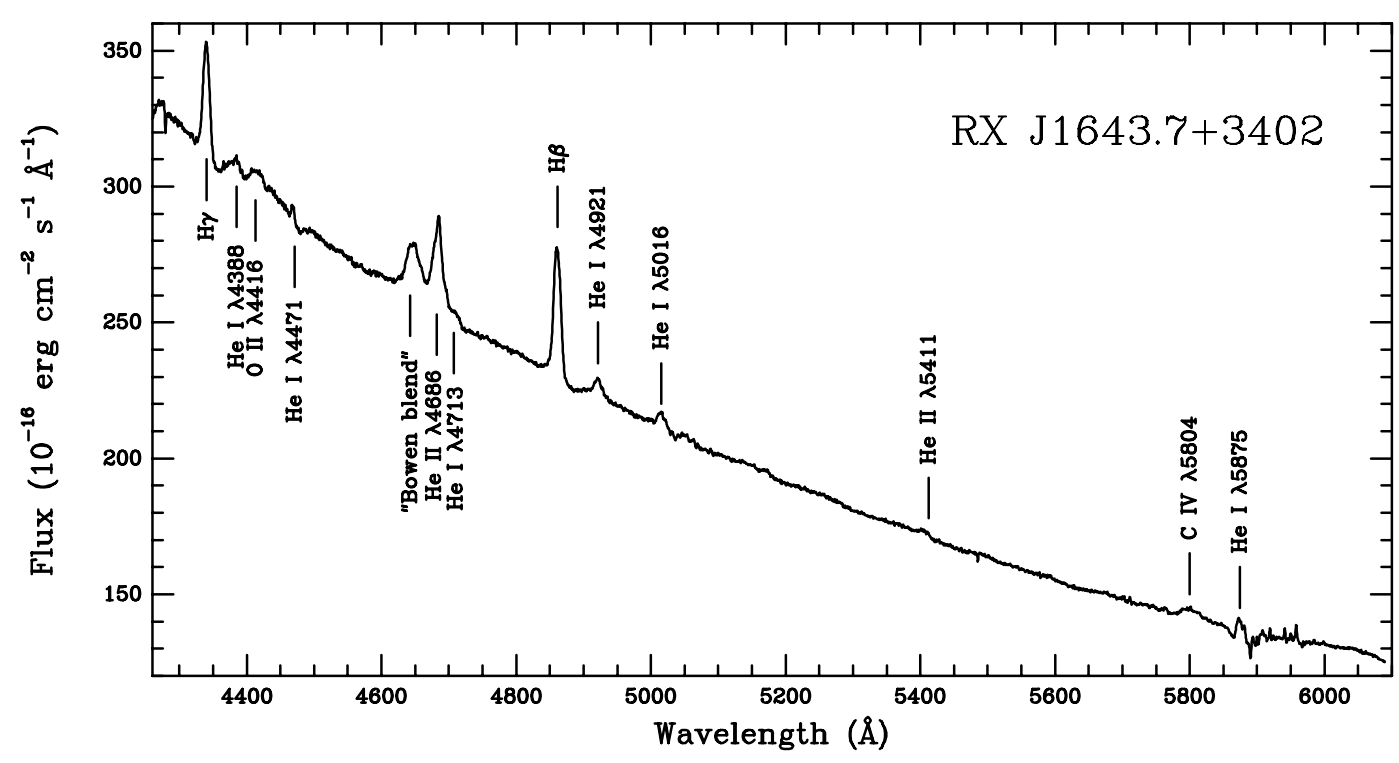

Fig. 2. Mean spectrum of RX J1643.7+3402, obtained at the OHP 1.93-m telescope with the CARELEC spectrograph, showing the blue continuum, approximately $F_{\lambda} \propto \lambda^{-2.7}$, and the main emission lines discussed in the text (the total exposure time is $387 \mathrm{~min})$.
Table 2. Magnitudes and colors of field stars shown in the finding chart for RX J1643.7+3402.

\begin{tabular}{cccccc}
\hline Name & $V$ & $U-B$ & $B-V$ & $V-R_{\mathrm{c}}$ & $R_{\mathrm{c}}-I_{\mathrm{c}}$ \\
\hline Star 2 & 12.97 & -0.03 & +0.54 & +0.32 & +0.26 \\
Star 3 & 12.16 & +0.94 & +1.04 & +0.61 & +0.44 \\
Star 4 & 14.74 & +0.89 & +1.08 & +0.59 & +0.48 \\
Star 5 & 16.30 & -0.21 & +0.43 & +0.34 & +0.29 \\
Star 6 & 16.68 & +0.04 & +0.66 & +0.39 & +0.31 \\
Star 7 & 16.43 & +1.18 & +1.56 & +1.02 & +1.01 \\
Star 8 & 16.14 & +0.55 & +0.87 & +0.46 & +0.38 \\
\hline
\end{tabular}

the two comparisons (stars 2 and 3, see Fig. 1). The calibration errors lie within \pm 0.02 and $\pm 0.05 \mathrm{mag}$, depending on the magnitude. Two different measurements of RX J1643.7+3402 were obtained for each color index, except $U-B$. The results are given in Table 3 . The rapid variability seen in the data of Fig. 3 may have slightly affected the derived colors since measurements in any pair of filters were taken one minute apart $(5 \mathrm{~min}$ in the case of the $U$ filter).

\section{Long-term variability}

We have investigated the long-term variability of this object by comparing the magnitudes derived from several Palomar Schmidt surveys. In the Guide Star Catalog GSC-ACT (Lasker et al. 1996-99), which is based on the "Quick-V" Survey (the epoch for the relevant plate is 1982.6), the GSC $V$ magnitudes for stars 2, 3 and 4 are 0.56 mag too bright with respect to the values in Table 2 and, correcting the catalog value for the variable, we derive $V=12.69$, well within the range seen in Fig. 3 .

For the POSS-I survey blue and red plates, the USNO A2 catalog (Monet et al. 1996) gives $O=15.2$ and $E=13.2$. While the blue magnitude is very much fainter than our values (by $3 \mathrm{mag}$ ), the Minnesota APS catalog (Pennington et al. 1993), also based on the POSSI survey, does not list either the variable or star 2 due
Table 3. Magnitudes and colors of RX J1643.7+3402 at two different times on 23 September 2000.

\begin{tabular}{lcccc}
\hline$V$ & $U-B$ & $B-V$ & $V-R_{\mathrm{c}}$ & $R_{\mathrm{c}}-I_{\mathrm{c}}$ \\
\hline 12.58 & -0.95 & -0.07 & +0.02 & -0.01 \\
12.65 & & -0.04 & +0.04 & +0.02 \\
\hline
\end{tabular}

to their proximity to the nearby bright star HD 151087. Thus we consider the USNO magnitudes for the variable to be unreliable (this is confirmed by fact that the USNO blue magnitude for star 2 is also too faint (by $1.3 \mathrm{mag}$ ), while the values for stars 3 to 8 differ little (by $-0.07 \mathrm{mag}$ ) from our $B$ magnitudes). In order to remedy this situation, we measured magnitudes for the variable and the stars of Table 2 on the Digital Sky Survey (DSS) POSS-I Red image (Epoch 1954.4) using our aperture photometry software and obtain $R_{\mathrm{c}}=12.7$ for the variable, consistent with our CCD photometry.

For the POSS-II survey, we measured magnitudes for the same stars using the DSS blue (epoch 1989.5) and red (epoch 1993.3) images and obtain $B=13.2$ and $R_{\mathrm{c}}=12.7$ for the variable. While the red magnitude is again in good agreement, the object appears fainter on the blue image by $0.6 \mathrm{mag}$.

We conclude that the brightness of RX J1643.7+3402 in the past does not appear to have undergone large variations relative to the values measured in September 2000.

\section{Period search}

\subsection{Radial velocities}

We analyzed the $\mathrm{H} \beta$ emission line velocities for periodic behavior using Discrete Fourier Transform (DFT) and Analysis-of-Variance (AoV) algorithms (as implemented in the MIDAS Time Series Analysis package). The short duration of the night runs (an average of 2.5 ), limited as it is in late September by hour-angle constraints, inevitably 


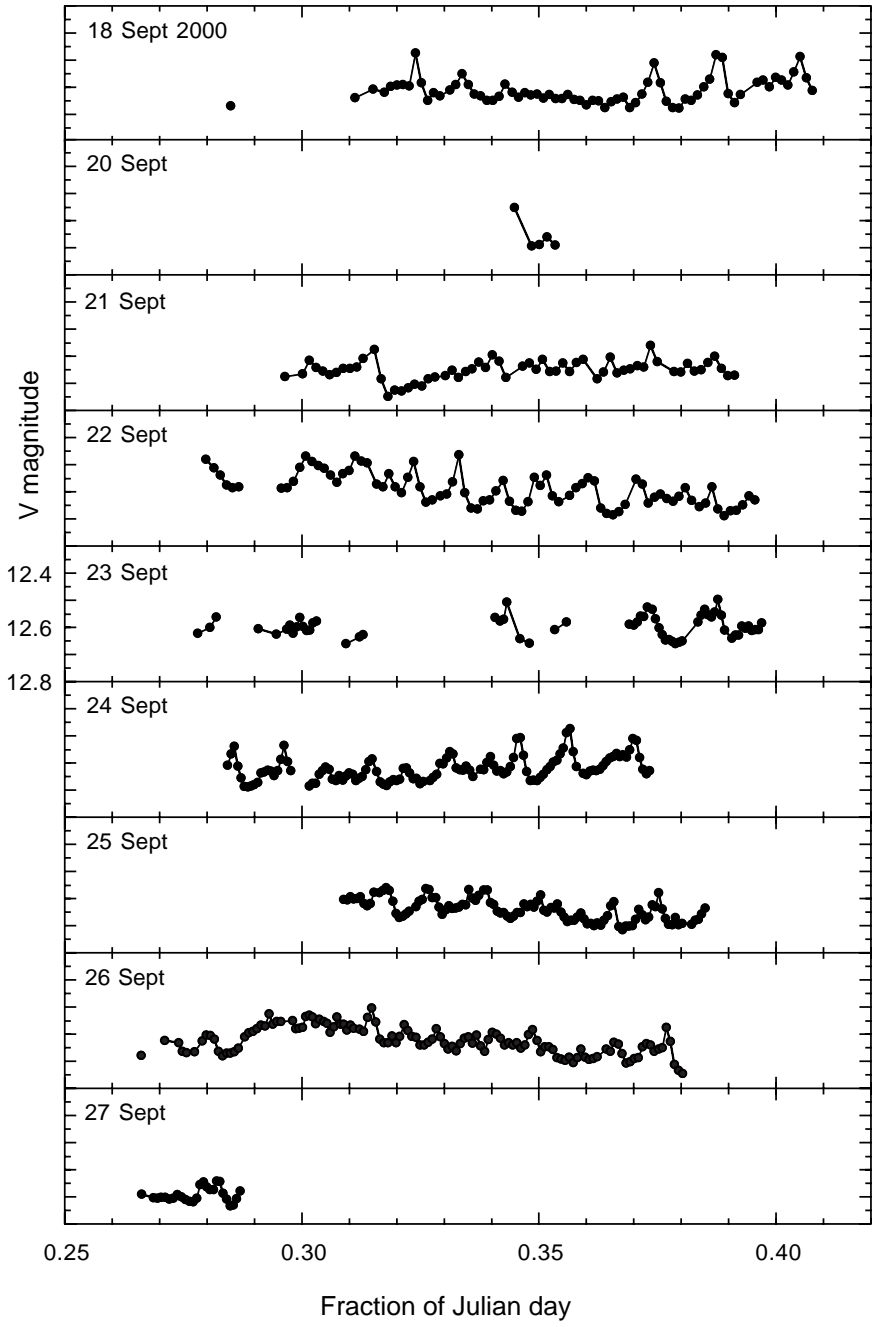

Fig. 3. CCD photometry of RX J1643.7+3402 obtained with the 1.2-m OHP telescope in the $V$ band on the dates shown. The magnitude scale is the same for all panels but is displayed only once. Note the strong ( 0.1 to $0.2 \mathrm{mag}$ ) rapid variations with a 15 min time scale which are present on most nights. The $\sim 0.1$ mag periodic modulation at $9.25 \mathrm{c} / \mathrm{d}(2.595)$ reported in the text can be discerned best on the nights of September 18, 22,24 and 26 .

introduces a window function with a large number of $1 \mathrm{c} / \mathrm{d}$ aliases for any existing period.

The raw DFT spectrum, computed using a frequency step of $0.01 \mathrm{c} / \mathrm{d}$, is shown in Fig. 4 for the interval 0-30 c/d. A more detailed plot is shown in Fig. 5 (thick dark curve) for the interval 4.5 to $12 \mathrm{c} / \mathrm{d}$ (periods from 5.3 to $2 \mathrm{~h}$ ). The strongest peaks are at $7.32,8.32$ and $9.32 \mathrm{c} / \mathrm{d}$. Removing the effects of the window function using the CLEAN algorithm (Roberts et al. 1987) produces a peak either at $8.32(2.885)$ or at $9.32 \mathrm{c} / \mathrm{d}(2.575)$, depending on the values used for the gain (the amount by which the window function is subtracted from the raw DFT at each iteration), number of iterations, and frequency step.

We also computed AoV periodograms (SchwarzenbergCzerny 1989) for the $\mathrm{H} \beta$ emission line radial velocity data using different numbers of phase bins and various amounts

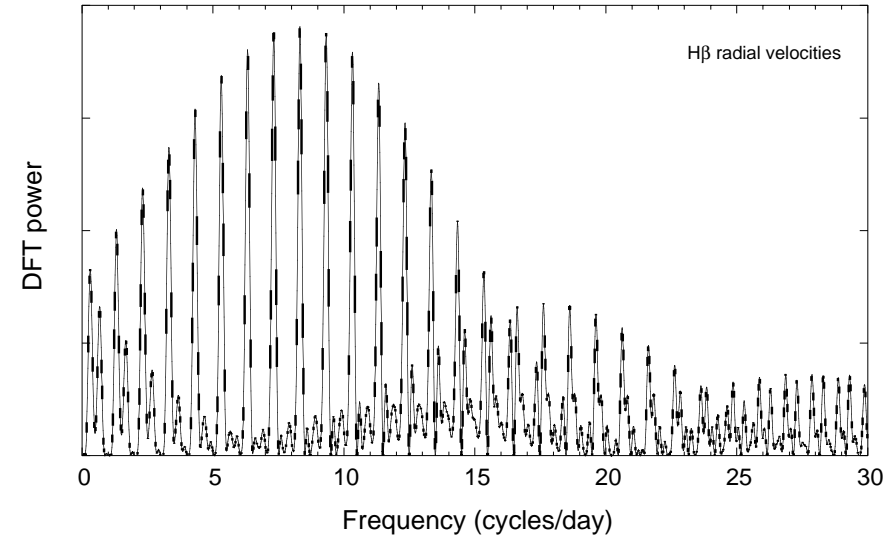

Fig. 4. The Discrete Fourier Transform (DFT) periodogram computed using 36 individual $\mathrm{H} \beta$ emission line radial velocities is displayed here for the frequency interval 0 to $30 \mathrm{c} / \mathrm{d}$. The strongest peaks are at $8.32 \pm 1 \mathrm{c} / \mathrm{d}$. The effects of the window function are apparent as aliases separated by $1 \mathrm{c} / \mathrm{d}$.

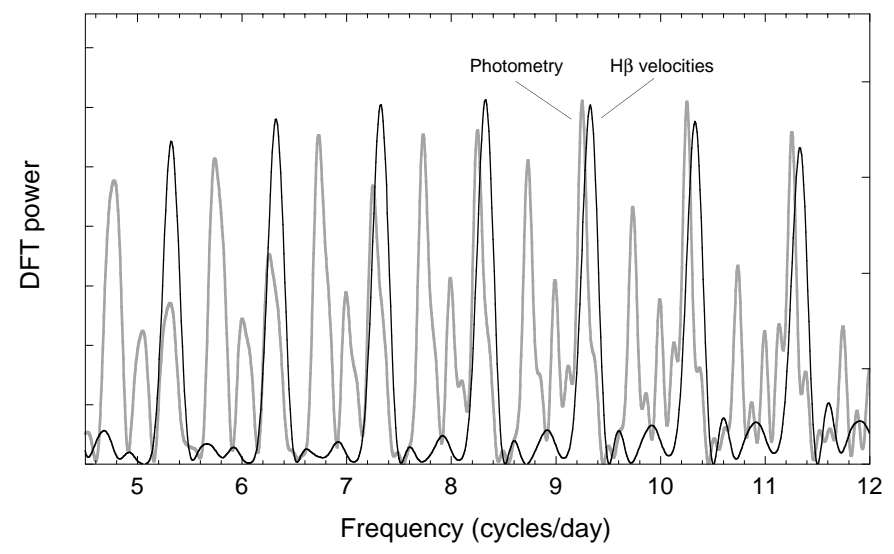

Fig. 5. DFT periodograms in the frequency interval 4.5 to $12 \mathrm{c} / \mathrm{d}$ for both the $\mathrm{H} \beta$ emission line radial velocities (dark curve) and the photometry (grey curve). The two curves have been normalized so that the strongest peaks in each appear of equal strength. The peaks in the velocity and the photometry periodograms appear at different frequencies.

of smoothing. In Fig. 6 we show the result for 8 bins and 3 overlapping bin covers (thick dark curve). The three strong peaks are at $8.32,9.32$ and $10.32 \mathrm{c} / \mathrm{d}$. The peak at $9.32 \mathrm{c} / \mathrm{d}(2.575)$ appears slightly stronger here, but the high-frequency noise present in the AoV periodogram, which arises from the small number of data points which move around from one phase bin to the next as the period changes, may mask the true strength of the peaks.

The results of our analysis are ambiguous. The existing radial velocity data appears insufficient to decide which of the two periods, $8.32 \mathrm{c} / \mathrm{d}(2.885)$ or $9.32 \mathrm{c} / \mathrm{d}(2.575)$, is the real orbital period of RX J1643.7+3402. A leastsquares sine fit to the velocities gives an estimate for the errors on the periods of $\pm 0.013 \mathrm{c} / \mathrm{d}( \pm 0.005)$.

The radial velocities are plotted in Fig. 7 as a function of phase (for the 2.575 period; the plot for the 2 h 885 period is nearly identical). The semi-amplitude is $K=97 \pm 12 \mathrm{~km} \mathrm{~s}^{-1}$. In the absence of any clear specific 


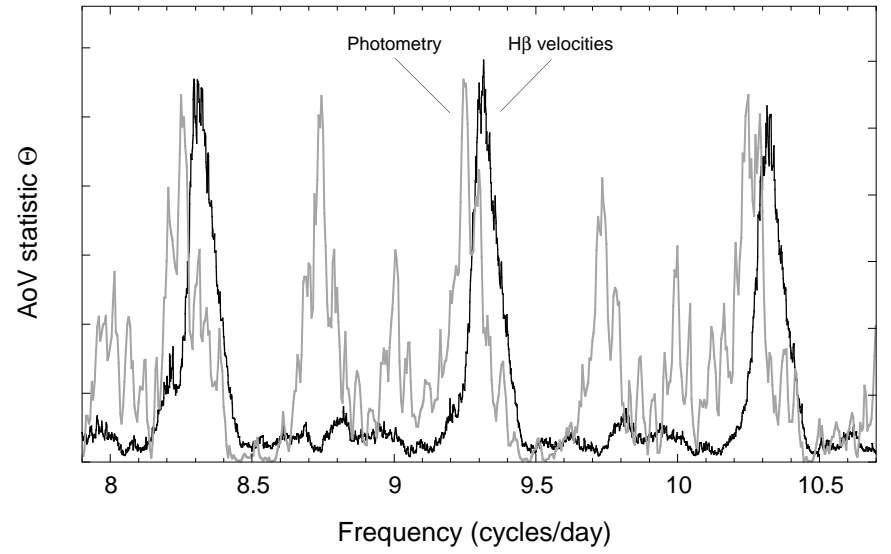

Fig. 6. AoV periodograms in the interval 7.9 to $10.7 \mathrm{c} / \mathrm{d}$ for both the $\mathrm{H} \beta$ radial velocities (dark curve) and the photometry (grey curve). The two curves have been normalized so that the strongest peaks in each appear of comparable strength. The peaks in the velocity and the photometry periodograms appear at different frequencies.

marker for orbital orientation, such as an eclipse, the epoch for phase 0 (HJD 2451806.0324 for the 2.575 period, HJD 2451806.1213 for the 2.885 period) has been defined as the time when the radial velocities change sign from negative to positive with respect to the heliocentric systemic velocity, which is $\gamma=-28 \pm 9 \mathrm{~km} \mathrm{~s}^{-1}$. Using this convention, and if the emission-line velocities reflect the motion of the white dwarf, which they may not, inferior conjunction of the white dwarf would take place at phase $\phi=0.0$.

\subsection{Light curves}

In order to search for the two possible periods detected in the radial-velocity data, and to look for a short-period modulation, we analyzed the photometric data with the DFT+CLEAN and AoV algorithms. We excluded from the analysis the very short nights of September 20 and 27, the night of September 23 which exhibits large gaps and the night of September 21 which, apart from a sharp discontinuity, appears flat and featureless. The selected set contains the data for September 18, 22, 24, 25 and 26, 502 images in all.

The resulting DFT periodogram was computed using a frequency step of $0.005 \mathrm{c} / \mathrm{d}$ and is shown in Fig. 5 (gray curve) together with the periodogram of the radial velocity data. It is also severely dominated by the window function but appears more complex (with two interleaved sets of peaks). The strongest peak appears at $9.25 \mathrm{c} / \mathrm{d}$ with an equally strong peak at $10.25 \mathrm{c} / \mathrm{d}$. The results of CLEANing the DFT spectrum show only a strong peak near $9.25 \mathrm{c} / \mathrm{d}(2.595)$, with a second weaker peak near $2.74 \mathrm{c} / \mathrm{d}(8.76)$ with one-half the power.

The results of an AoV analysis of the photometry are also shown in Fig. 6 (grey curve), together with the periodogram of the radial-velocity data. The $9.25 \mathrm{c} / \mathrm{d}$ peak appears stronger than the peak at $10.25 \mathrm{c} / \mathrm{d}$, and also

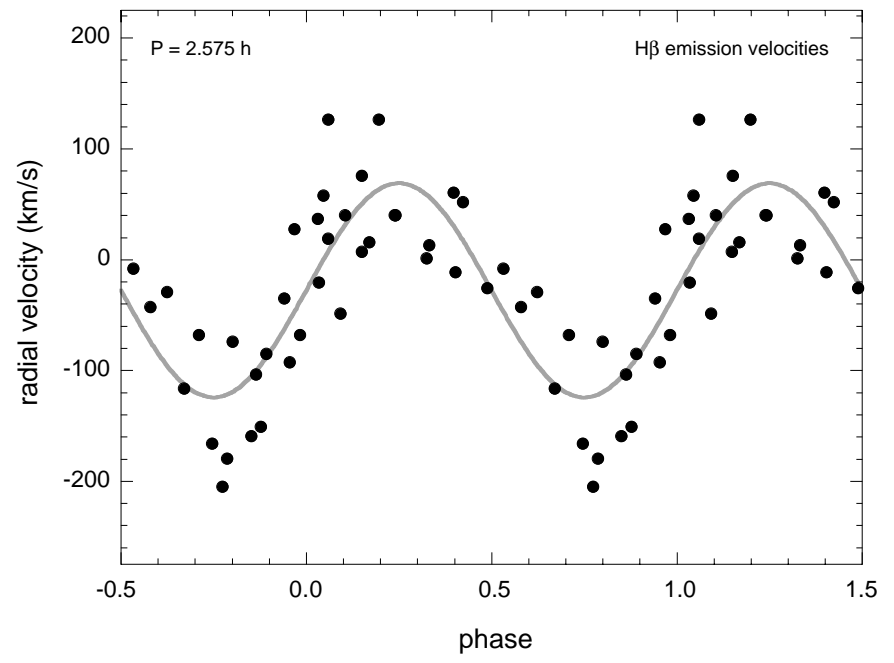

Fig. 7. $\mathrm{H} \beta$ emission line radial velocities folded using the $9.32 \mathrm{c} / \mathrm{d}(2.575)$ period. The epoch for phase 0.0 is HJD 2451806.0324. The semi-amplitude is $K=97 \pm 12 \mathrm{~km} \mathrm{~s}^{-1}$ and the heliocentric systemic velocity is $\gamma=-28 \pm 9 \mathrm{~km} \mathrm{~s}^{-1}$. The curve for the other possible spectroscopic period, $8.32 \mathrm{c} / \mathrm{d}$ $(2.885)$, is nearly identical.

stronger than the peak at $8.25 \mathrm{c} / \mathrm{d}$. The peaks in Figs. 5 and 6 derived from the photometry analysis have frequencies $(8.25$ and $9.25 \mathrm{c} / \mathrm{d})$ that are similar to, but different from, those found from the analysis of the radial velocity data $(8.32$ and $9.32 \mathrm{c} / \mathrm{d})$. The difference is small $(0.8 \%)$ but significant.

When the differential photometry data for individual nights are folded using one of the spectroscopic periods, a modulation is seen which clearly shifts forward in phase from night to night, but when the data are folded using the closest photometric period, the modulations do not shift and are in phase with each other. The results for the selected subset of nights (September 18, 22, 24, 25 and 26) are shown in Fig. 8 folded using one of the possible photometric periods (the others yield similar curves). Although small (1 to $2 \%$ ) night-to-night changes in mean level are present, no corrections have been applied to the data plotted in this figure. There is a significant average modulation of 0.1 mag peak-to-peak amplitude at $9.25 \mathrm{c} / \mathrm{d}(2.595)$ but little or none is seen at $9.32 \mathrm{c} / \mathrm{d}(2.575)$, the value found from the radial velocities. The modulation can be discerned best in the light curves of Fig. 3 during the nights of September 18, 22, 24 and 26.

In summary, one likely interpretation of our results is that the radial velocities are modulated with a $9.32 \mathrm{c} / \mathrm{d}$ $(2.575)$ period while the photometric data show a sligthly longer $(0.8 \%)$ period of $9.25 \mathrm{c} / \mathrm{d}(2.595)$. However, an alternative interpretation where the radial-velocity data are modulated with a $8.32 \mathrm{c} / \mathrm{d}(2.885)$ period while the photometric data display a noticeably shorter $(10 \%)$ period of $9.25 \mathrm{c} / \mathrm{d}(2 \mathrm{~h} .595)$ is also possible. Note that the other photometric periods (at $\pm 1 \mathrm{c} / \mathrm{d}$ ) are not entirely excluded. The implications of these two different interpretations are discussed later. 


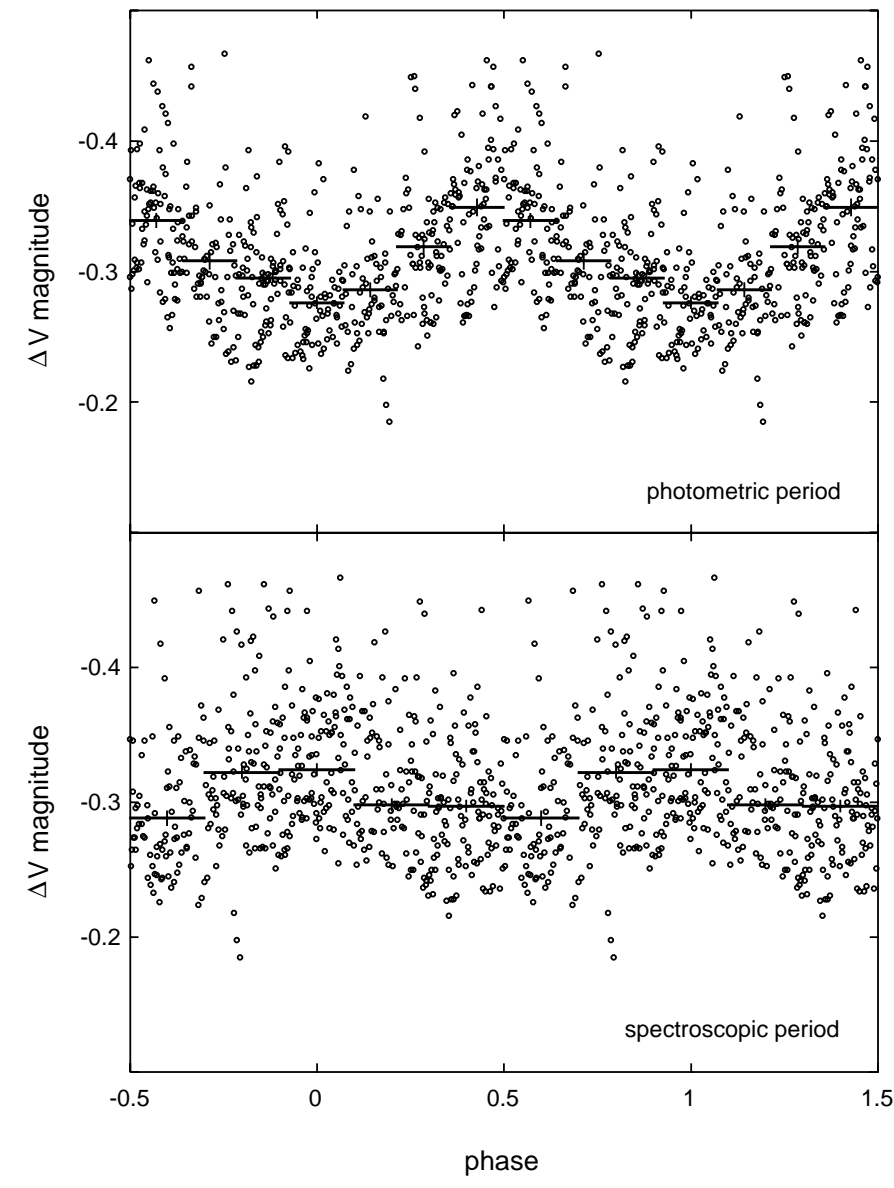

Fig. 8. CCD $V$-band differential light curves constructed by folding the individual measurements for a selected subset of nights (September 18, 22, 24, 25 and 26) using one set of possible periods (a photometric period at $9.25 \mathrm{c} / \mathrm{d}$ ( or 2.595$)$ and a spectroscopic period at $9.32 \mathrm{c} / \mathrm{d}$ ( or 2.575$)$ ). The curves for the other possible periods are similar. No corrections for the small night-to-night changes in mean level have been applied here. Epoch for phase 0 is HJD 2451806.036 . The magnitudes are expressed with respect to star 2. The data are also shown averaged into 7 and 5 bins, respectively, with the corresponding errors on the mean values. The peak-to-peak amplitude in the upper panel is $\sim 0.1 \mathrm{mag}$.

\subsection{Rapid variability}

None of our nights was sufficiently long to do a reliable time analysis within a single night for periods around 15 min and we looked instead for a coherent period in our data by grouping the longest nights with uninterrupted coverage which clearly displayed the rapid variability (September 18, 22, 24, 25, 26) and subjected them to an AoV analysis (within the $50-150 \mathrm{c} / \mathrm{d}$ interval) using three phase bins and no smoothing. There is no single peak which stands out in the resulting AoV periodogram, but the strongest signals are located between $80-85 \mathrm{c} / \mathrm{d}$ (18-17 min) and around $100 \mathrm{c} / \mathrm{d}(14 \mathrm{~min})$, confirming the visual impression of a time scale around $15 \mathrm{~min}$. The CLEANed DFT spectrum agrees with these results, showing power principally near $84.2 \mathrm{c} / \mathrm{d}$ and at $101.8 \mathrm{c} / \mathrm{d}$, with the latter peak being the strongest.

\section{Spectroscopic characteristics}

\subsection{Balmer and He I lines}

The profiles of all emission lines are complex and variable. A few of the $\mathrm{H} \beta$ profiles show a strong, narrow absorption; but most have a flat top or a shoulder, suggesting the presence of weak absorption in the line core. In addition, some of the profiles show weak blue emission, and/or red absorption. We fitted each of the $\mathrm{H} \beta$ profiles with several Gaussian components (see Fig. 9): (1) an emission component, the "main" component, (2) a narrow absorption component (assumed to be unresolved and thus assigned a fixed width of $4.0 \AA F W H M$ ), (3) a blue emission component and (4) a broad red absorption component. Due to their location in the line wings and to their relative weakness the two latter components were constrained, the first in width (fixed at $8.0 \AA F W H M$ ) and the second in width (fixed at $35 \AA F W H M$ ) and in velocity (fixed at $\left.+135 \mathrm{~km} \mathrm{~s}^{-1}\right)$.

We find that the main component has a variable $F W H M(8.0-15.0 \AA)$, but half of the values are in the range $10.6-11.8 \AA$. The analysis of $\mathrm{H} \gamma$ and $\mathrm{He} \mathrm{I} \lambda 5875$ gives similar results; the velocities of the main component of the three lines are well correlated. The $\mathrm{H} \beta$ and $\mathrm{H} \gamma$ FWHMs are also correlated, suggesting that the observed variability is real and not the result of measurement errors. We also note that the presence, around phases $\phi \approx 0.8-0.2$, of a blue emission and of a red absorption in $\mathrm{H} \beta$ are well correlated in the sense that, when a red absorption is present, a blue emission is always present; moreover, we never observe a red emission nor a blue absorption.

The velocities of the narrow Gaussian absorption component fitted to the $\mathrm{H} \beta$ profiles (see Fig. 9) were measured to have a semi-amplitude $K=107 \pm 19 \mathrm{~km} \mathrm{~s}^{-1}$ and an average velocity $\gamma=+6 \pm 13 \mathrm{~km} \mathrm{~s}^{-1}$. They follow closely the main $\mathrm{H} \beta$ emission component as there is no significant phase shift $(0.035 \pm 0.034)$. The intensities of the main Gaussian component fitted to the data are seen to vary in phase with a minimum in the phase range 0.9-0.3 and a maximum near $0.6-0.8$. The high-velocity blue emission was measured on 15 spectra and its velocities give a semiamplitude $K=280 \pm 110 \mathrm{~km} \mathrm{~s}^{-1}$ and an average velocity $\gamma=-890 \pm 76 \mathrm{~km} \mathrm{~s}^{-1}$. The phase of maximum blue shift is $0.95 \pm 0.06$. There is one spectrum where the velocity was measured to be $-1880 \mathrm{~km} \mathrm{~s}^{-1}$, but others lie within the interval -650 to $-1200 \mathrm{~km} \mathrm{~s}^{-1}$. The curve of the $\mathrm{H} \beta$ highvelocity component shows a phase lag of 0.2 with respect to the main emission.

\section{2. "Bowen blend" and $\mathrm{He} / / \lambda 4686$}

The "Bowen blend" is often observed in binary X-ray sources (McClintock et al. 1975); in Sco X-1, its constituents are N III $\lambda \lambda 4634,4641$ and 4642 and C III $\lambda \lambda 4647,4651$ and 4652 ; the N III lines account for about two-thirds of the total blend intensity; the theoretical ratio $I(\lambda 4634) / I(\lambda \lambda 4641,4642)$ is 0.71 (Schachter et al. 1989). 


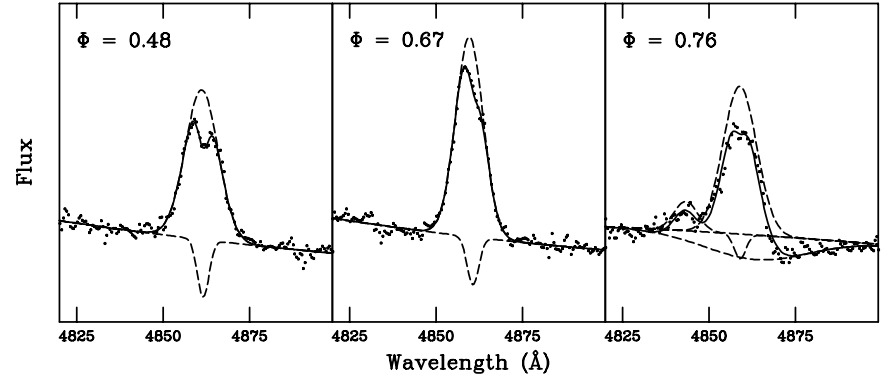

Fig. 9. Three examples of the fit of the $\mathrm{H} \beta$ feature with Gaussian components. The leftmost panel shows a profile with a clear narrow absorption component. The middle panel show the case of a profile displaying a "shoulder", accounted for by a red-shifted absorption component. The right panel shows a case with a "flat-topped" profile where both blue emission and red absorption components were needed for a good fit. The spectra have been normalized through division by the continuum.

The N III lines are produced by fluorescence, the C III lines are not (Bowen 1935; Ferland 1992).

We analysed the spectral region around He II $\lambda 4686$, which contains the "Bowen blend", by using six Gaussian profiles: the first two, intended to represent the $\mathrm{N}$ III fluorescent lines, were centered at 4634.2 and $4641.0 \AA$ respectively; they were forced to have the same width and velocity; the flux of the first was assumed to be half that of the second; the third profile, centered at $4649.0 \AA$, corresponded to the C III triplet; two more profiles were judged necessary to fit reasonably the He II line itself; the sixth component corresponds to He I $\lambda 4713.1$; we fixed its width to $12 \AA(F W H M)$, the average width found for the Balmer main emission components. The fit yielded a very weak, insignificant N III system in emission, suggesting that, in RX J1643.7+3402, the C III triplet accounts for most of the "Bowen blend". The average FWHM of both the C III triplet at $\lambda 4650$ and the C IV doublet at $\sim \lambda 5804$ is about $1500 \mathrm{~km} \mathrm{~s}^{-1}$.

The Gaussian fits to the He II emission line required two different components, a "broad" component and a "narrow" component, examples of which are illustrated in Fig. 10. The velocities of the "broad" component vary between 0 and $-350 \mathrm{~km} \mathrm{~s}^{-1}$. The semi-amplitude is $K_{\mathrm{b}}=186 \pm 25 \mathrm{~km} \mathrm{~s}^{-1}$ and the mean velocity is $\gamma_{\mathrm{b}}=-197 \pm 18 \mathrm{~km} \mathrm{~s}^{-1}$. The phase lag with respect to the velocities of the main $\mathrm{H} \beta$ emission component is very small $(0.054 \pm 0.030)$ and may in fact be negligeable. The "narrow" He II component shows a curve similar to the "broad" component but is shifted in velocity by $\sim+300 \mathrm{~km} \mathrm{~s}^{-1}$ (see Fig. 12); this suggests that these two components probably describe a complex single profile. Two individual spectra show a particularly striking redshifted "narrow" component (see Figs. 10 and 12) near phase 0.75 . Phase coverage for the "narrow" component is uneven since none is seen in some spectra (phase 0.42) while in others its absence may in part be due to a poor $S / N$ ratio.

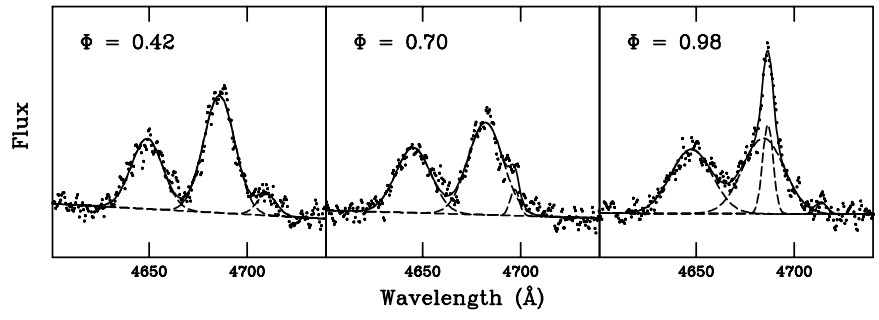

Fig. 10. Three examples of the fit of the He II $\lambda 4686$ line and the "Bowen blend" with Gaussian components. The spectra have been normalized by dividing them by the continuum. The "Bowen blend" has been fitted by a combined C III feature at $\lambda 4649$; the velocity and width of the C III line has been forced to be equal to that of the broad He II line. In the left panel there is no He II narrow component, but the He I $\lambda 4713$ line is visible. In the middle panel there is a He II red-shifted narrow component, while in the right panel the He II line has a strong narrow component.

\section{3. "Trailed" spectra}

We constructed "trailed" spectra by folding all the normalized spectra into 10 phase bins using the 2.575 period. The spectra were then smoothed in wavelength using a 3-point running average and in phase using a $10 \times$ 1 Gaussian filter. The result is displayed using 20 phase bins. Figure 11(top panel) shows three sections of this spectrum (each panel is $140 \AA$ wide) including respectively $\mathrm{H} \beta$ and He I $\lambda 4921$, the "Bowen blend" and He II $\lambda 4686$, and C IV $\lambda 5804$ and He I $\lambda 5875$; all narrow lines $(\mathrm{H} \beta$, He I and He II) show a clear $S$-shape. The He I $\lambda 5875$ line has three main components: an emission component behaving like $\mathrm{H} \beta$, a broad absorption component and a narrow (unresolved?) absorption component visible mainly at phases $\sim 0.3-0.6$. The $\mathrm{H} \beta$ line (shown twice with different contrasts) has a strong emission core with a narrow absorption component (barely visible in the figure) which is most conspicuous at the same phases as in the He I line; in addition, a very weak broad blue emission appears during about half the period, while a broad red absorption is detected simultaneously. The He II line has a narrow core similar to the $\mathrm{H} \beta$ core, but without any trace of the narrow absorption component; in addition, there is a broad emission component.

The bottom panel of Fig. 11 shows the result of subtracting the phase-averaged profile from the "trailed" spectra shown in the top panel. This enhances the relative changes in the emission lines. Complex low-velocity modulations, which have a distinct "braided" appearance, are seen in the Balmer lines, the He I lines and the He II line. The modulations in the Balmer and He I lines are similar, although the He I $\lambda 5875$ line profile is complicated by the stronger central absorption. In the case of the He II $\lambda 4686$ line there is an additional blue-shifted highvelocity $S$-wave which is clearly visible for roughly onehalf of the orbital cycle, from phases 0.6 to 0.2 , roughly opposite to the time when the central absorption is strongest in the Balmer and He I lines. This blue-shifted He II 

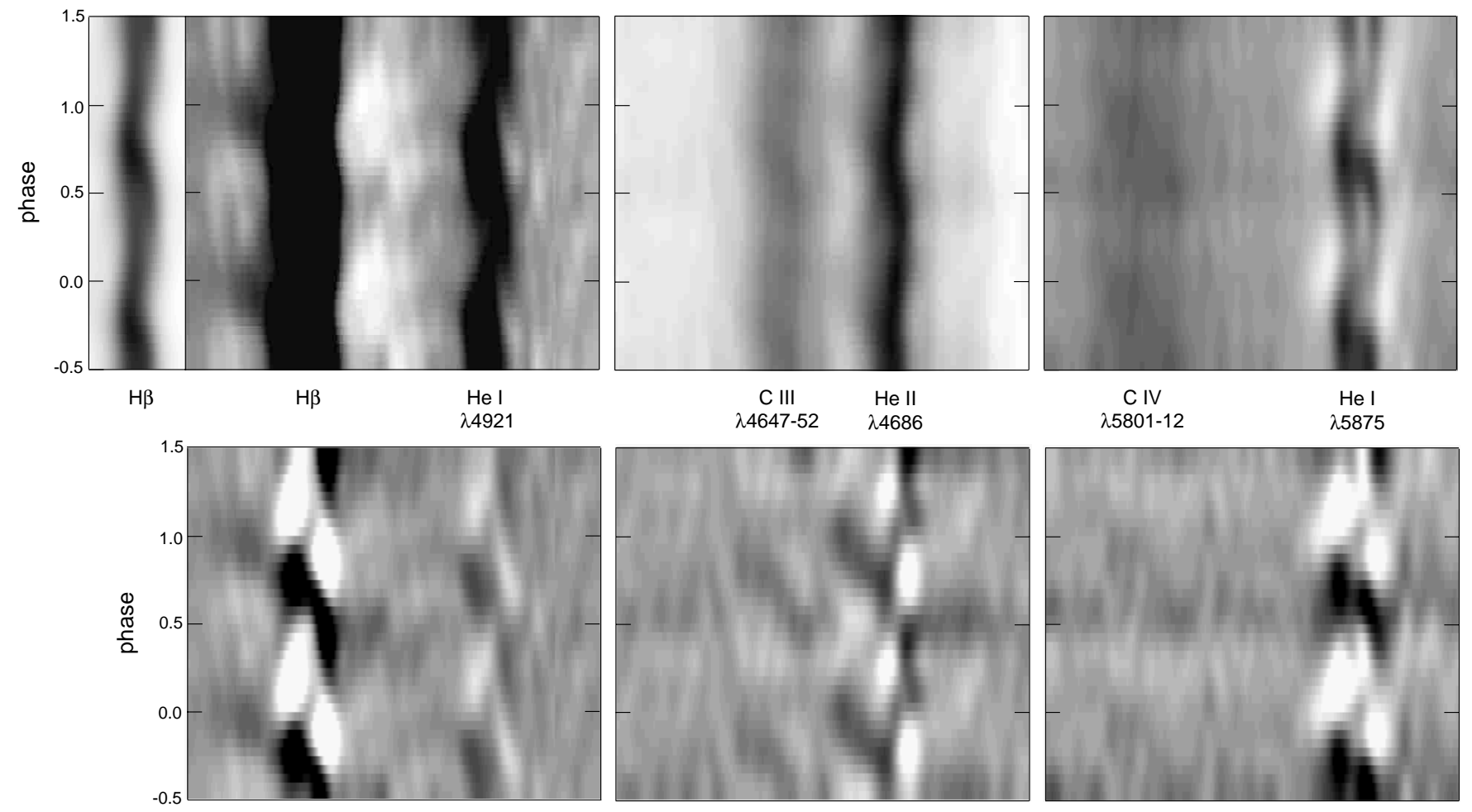

Fig. 11. Top panel: "trailed" spectra built by folding all normalized spectra into ten phase bins assuming a period of 2.575 . The spectra were then smoothed as described in the text. The result is displayed using 20 phase bins. All narrow lines (H $\beta$, He I and $\mathrm{He}$ II) have a sinusoidal shape; the $\mathrm{H} \beta$ line is shown twice with different contrasts; the He I $\lambda 5875$ line clearly shows a central absorption component which is most conspicuous at phases $\sim 0.3-0.6$. The $\mathrm{Na} \mathrm{D}$ absorption lines to the red of He I $\lambda 5875$ did not display any detectable phase shift and have been numerically removed to reveal the red He I absorption components. Bottom panel: the residuals obtained by substracting the phase-averaged profile from the "trailed" spectra shown in the top panel. This enhances the relative changes in the emission lines as a function of phase. Complex low-velocity modulations, having a distinct "braided" appearance, are seen in the Balmer lines, the He I lines and the He II line. Note the high-velocity blue-shifted He II $S$-wave visible for one-half of the cycle. The wavelength interval shown for each panel is $140 \AA$.

emission $S$-wave also lags in phase by 0.2 with respect to the "main" profile, showing roughly the same velocity curve as the blue-shifted $\mathrm{H} \beta$ emission component.

There is a weakly visible $S$-wave both in the "Bowen blend" and an even weaker one in the C IV blend which is seen in the phase range from 0.4 to 0.9 , both moving in sympathy with the blue-shifted He II $S$-wave. The He I $\lambda 5875$ line displays blue-shifted absorption when both the $\mathrm{H} \beta$ and the He II emission show blue-shifted emission. In Fig. 12 we have plotted the radial velocities of the He II $\lambda 4686 S$-wave, as measured on the residual "trailed" spectra, together with the velocities of the two components ("broad" and "narrow") of the "main" emission discussed earlier.

\section{Discussion}

\subsection{What kind of system?}

The blue continuum, the relatively weak, high excitation emission lines and the relative long-term stability in brightness suggest that RX J1643.7+3402 belongs to the novalike class of cataclysmic variables (CVs). These objects are believed to transfer mass at a rate high enough so that they are stuck permanently in outburst, thus preventing the instabilities associated with dwarf novae. The spectrum shown in Fig. 2 resembles that of V795 Her (Casares et al. 1996; Dickinson et al. 1997), DW UMa (Shafter et al. 1988), LX Ser (Young et al. 1981) and V442 Oph (Hoard et al. 2000) and looks very similar to that of V533 Her (Thorstensen \& Taylor 2000). All these systems belong to the SW Sex sub-group of novalike CVs. The distinctive characteristics of SW Sex stars are (1) single-peaked emission lines, particularly He II $\lambda 4686$, instead of the doublepeaked lines typical of a Keplerian disk, (2) gross asymmetries in the emission lines from the disk, so that they do not reflect the motion of the white dwarf, (3) about two-thirds of the systems (11 out of 16) display eclipses of the accretion disk by the low-mass late-type companion, (4) peculiar absorption features in most lines at a phase opposite the eclipse, near maximum blueshift of the Balmer emission lines, (5) frequently, high-velocity $S$-waves in the Balmer emission lines, and (6) orbital periods grouped mostly in the 3-4-h range, but with two objects in the period "gap" and one having an 8-h period. The two SW Sex stars with the shortest periods, V442 Oph $(P=2.98)$ and V795 Her $(P=2.60)$ are non-eclipsing systems (Hoard et al. 2000; Casares et al. 1996). 


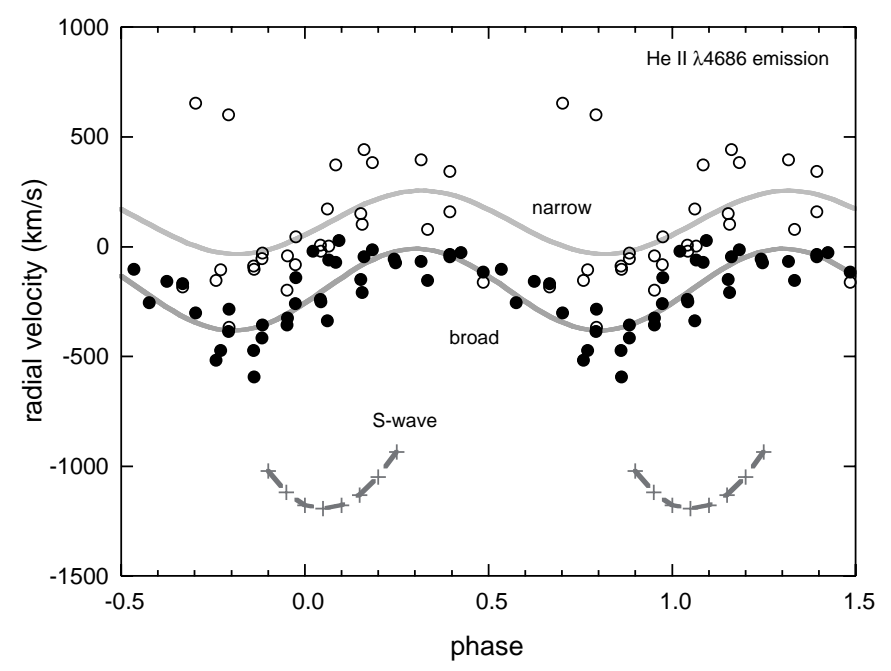

Fig. 12. He II $\lambda 4686$ radial velocities are shown for the two components of the "main" emission ("broad" and "narrow") and for the blue $S$-wave. The period and epoch used are the same as for Fig. 7. For the "main" components we show the least-squares sine fits. The semi-amplitudes are $K_{\mathrm{b}}=186 \pm$ $25 \mathrm{~km} \mathrm{~s}^{-1}$ and $K_{\mathrm{n}}=144 \pm 65 \mathrm{~km} \mathrm{~s}^{-1}$ and the average velocities are $\gamma_{\mathrm{b}}=-197 \pm 18 \mathrm{~km} \mathrm{~s}^{-1}$ and $\gamma_{\mathrm{n}}=+110 \pm 52 \mathrm{~km} \mathrm{~s}^{-1}$. For the $S$-wave component the velocities were measured on the residual "trailed" spectra shown in Fig. 11 (bottom panel).

The "trailed" spectra of Fig. 11 show phase-dependent absorption reminiscent of that characterizing the SW Sex stars (see Thorstensen et al. 1991). However, the absorption we see is strongest in the phase range $0.3-0.6$, near the positive-to-negative cross-over point for the $\mathrm{H} \beta$ emissionline velocities, whereas in SW Sex stars the absorption is normally strongest near the phase of maximum approach velocity, approximately one-half cycle away from the eclipses seen in most of these systems. As no eclipses have been detected in our photometry we cannot determine the relation between the radial velocities and the binary system orientation, but the relative difference in the times of appearance of the strongest absorption in SW Sex type stars and in our object, roughly one-quarter of an orbital cycle, is very large and does not depend on any particular phase convention. The $U B V$ colors measured for RX J1643.7+3402 are very similar to those reported for the non-eclipsing systems V442 Oph and V795 Her (see the compilation by Hoard 1998), perhaps a consequence of their accretion disks being viewed at lower inclinations.

There is currently no single agreed-upon explanation for the SW Sex phenomenon, but it appears that the accretion disks of these systems have a complicated structure. For example, the flared-out disk in DW UMa (Knigge et al. 2000) masks parts of the disk surface at any given time and can thus explain the gross asymmetries and the single-peaked profiles of the emission lines (with an additional contribution from a disk wind) while substantial stream-disk overflow (Hellier 1998) may explain the absorption in the line cores, when the gas stream is seen projected onto the disk surface. The high-velocity $S$-wave emission features may be due to stream re-impact in an area near the disk center, where the Keplerian velocities are highest (Hellier \& Robinson 1994). The detailed interpretation of these $S$-waves is under debate. Casares et al. (1996) consider the strong red and the weak blue wings in the V795 Her system as two separate components, perhaps related to accretion onto a magnetic white dwarf which is rotating synchronously, while Dickinson et al. (1997) explain the two wings of that system as parts of a very broad emission $S$-wave with a sympathetically phased red-shifted superimposed absorption $S$-wave. In RX J1643.7+3402 only the blue wing is seen in emission. The $\mathrm{H} \beta$ profile shown in the right panel of Fig. 9 is similar to those observed in V795 Her.

The role of magnetic fields in explaining the characteristics of SW Sex stars has been discussed, among others, by Horne (1999) who introduced the idea of a diskanchored magnetic propeller. More recently, the discovery of variable circular polarization in LS Peg (Rodriguez-Gil et al. 2001) indicates that magnetic accretion plays an important role in these systems. They suggested that SW Sex systems may just be intermediate polars with high accretion rates.

RX J1643.7+3402 shares several characteristics with SW Sex stars, such as single-peaked instead of doublepeaked emission lines, asymmetric profiles, high-velocity $S$-waves (although in this system we only see the blue part of this $S$-wave) and absorption features in the line cores. It has, in particular, many properties in common with V795 Her, including similar spectroscopic period, rapid photometric behavior and superhump modulation. The 0.2 phase lag we find between the high-velocity $S$-wave and the main emission component for both $\mathrm{He}$ II and $\mathrm{H} \beta$ has also been found in the SW Sex system V1315 Aql (Hellier 1996). This phase lag has been interpreted as arising from the difference in the location, within the disk, of the two emission regions: the high velocity component coming from the inner disk (re-impact area?) and the lower velocity component from a more external location (hot spot?). The phasing of the absorption features in RX J1643.7+3402, which are strongest when the main emission velocities change from recession to approach, is nevertheless different from that in SW Sex systems, where they appear close to the time of maximum approach velocity, suggesting a different geometry in the accretion stream disk-overflow.

\subsection{Optical modulation}

In the section on the period search we have seen that the exact value of the spectroscopic period of this system is not yet known and may be either 2.575 or 2.885 , both within the period "gap" (Shafter 1992), which although sparsely populated, contains several novalike systems. The photometric modulation detected on most nights (of $\sim 0.1$ mag amplitude in $V$ ) at a probable period of 2.595 (but the $\pm 1 \mathrm{c} / \mathrm{d}$ aliases are not excluded), different from either of the spectroscopic periods, could be explained as a 
superhump modulation. In many dwarf novae, but also in novalike variables, photometric modulations are seen with a period different from the spectroscopic period (Taylor et al. 1998). This phenomenon is known as the superhump phenomenon and corresponds to the light variations of a precessing elongated accretion disk. In the case of dwarfnovae the superhumps appear only in outburst but, in novalike systems, the phenomenon is persistent and has been called "permanent" superhumps. While most superhump periods are longer than the orbital period ("positive" superhumps), some appear at a shorter period ("negative" superhumps). It has been suggested that the former arise from the prograde motion of the line of apsides of an elongated disk, while the latter appear to imply a tilted, elongated disk displaying retrograde motion of the line of nodes (Patterson et al. 1997).

Patterson (1999) has reported that among a sample of 18 non-dwarf nova systems, 7 show positive superhumps, 4 show negative superhumps and 7 show both kinds. Essentially all novalike systems with $P_{\text {orb }}<3$-h show positive superhumps. Only novalike systems show negative superhumps and, amongst these, four are SW Sex systems. Patterson also shows that the fractional period excess $\epsilon=\left(P_{\mathrm{sh}}-P_{\text {orb }}\right) / P_{\text {orb }}$ shows a correlation with orbital period, with negative superhumpers showing a period excess about one-half that for positive superhumpers. For the spectroscopic periods reported here, these correlations would predict $\epsilon \sim+0.07$ for a positive superhump and $\epsilon \sim-0.03$ for a negative one. The values we apparently find, +0.008 or -0.10 , are significantly different. Further observations will reveal the true orbital period of RX J1643.7+3402 and should allow a more detailed characterization of the optical modulation.

\subsection{Rapid variability}

While rapid variability is typical of most cataclysmic variables, the behavior we see in this object seems unusual, since the variations show a large amplitude ( 0.1 to $0.2 \mathrm{mag}$ ) and each peak in brightness seems well separated from its neighbor. Rapid variations of a similar character have been reported in the case of V795 Her (Zhang et al. 1991). These variations have been variously interpreted in the literature as (1) oscillations of the disk boundary layer, (2) reprocessing of X-rays in "blobs" of optically thick material orbiting within the accretion disk, and (3) accretion of "blobs" onto the magnetic poles of a magnetized white dwarf. Longer observing runs are needed to obtain more accurate information on the characteristics of the rapid variations in RX J1643.7+3402.

\subsection{Distance and X-ray luminosity}

The average value for the $E W$ of the $\mathrm{H} \beta$ emission line for this object is $2.8 \AA$. The correlation between the absolute visual magnitude and the $\mathrm{H} \beta E W$ derived by Patterson (1984) for cataclysmic variables suggests that the absolute magnitude might be brighter than $M_{V}=+6$. Assuming negligeable absorption, this yields a lower limit for the distance of 210 pc. For novalike systems Warner (1995) discusses the correlation between absolute visual magnitude and orbital period. For the period reported here this correlation yields $M_{V}=+5 \pm 1$, consistent with the previous estimate. Since these values refer to a standard inclination angle of $\sim 57^{\circ}$, the correction in this case is probably small since no eclipses are detected. The entry in the ROSAT bright source catalogue gives a PSPC $(0.1-2.4 \mathrm{keV})$ count rate of $0.05 \pm 0.01$ count $^{-1}$ and a hardness ratio HR 1 of $0.62 \pm 0.22$. Using the conversion factor given by Fleming et al. (1995) the X-ray flux is $7.1 \times 10^{-13} \mathrm{erg} \mathrm{cm}^{-2} \mathrm{~s}^{-1}$. We note that V795 Her has also been detected by ROSAT at approximately the same flux level (Rosen et al. 1995). Assuming the distance derived above and no X-ray absorption this yields a very approximate $0.1-2.4 \mathrm{keV}$ X-ray luminosity of $3.8 \times 10^{30} \mathrm{erg} \mathrm{s}^{-1}$, which is near the lower end of the range observed for CVs (van Teeseling \& Verbunt 1994; van Teeseling et al. 1996). The X-rays may be produced in the boundary layer between the white dwarf and the accretion disk but more X-ray data are needed to investigate the origin of the emission.

\section{Conclusion}

We have reported the discovery of a new bright $(V \sim 12.6)$ cataclysmic variable star identified with the ROSAT X-ray source RX J1643.7+3402. Spectroscopic and photometric observations show it to be a novalike cataclysmic variable sharing several of the characteristics of the SW Sex subclass of novalike CVs. The spectroscopic period may be either $2^{\mathrm{h}} .575$ or $2^{\mathrm{h}} 885$, both within the period "gap". A photometric modulation (of $\sim 0.1$ mag amplitude in $V$ ) with a probable period of 2.595 is present on most nights and could be either a "positive" or a "negative" superhump modulation (depending on the exact spectroscopic period), indicating the presence of a precessing accretion disk in this system. Rapid variations of 0.1 to $0.2 \mathrm{mag}$ amplitude in $V$ repeat with a time scale of $15 \mathrm{~min}$. More observations are needed to resolve the spectroscopic period ambiguity, to study the optical modulation and refine its period and to fully characterize the rapid variations.

Acknowledgements. This work was supported by the JUMELAGE "Astrophysique France-Arménie", a bilateral program funded by the French Centre National de la Recherche Scientifique and the French Ministère des Affaires Étrangères. We wish to thank Robert Rutledge and Pablo Rodríguez-Gil for their comments on the manuscript. The Guide Star Catalog and the Digitized Sky Survey were produced at the Space Telescope Science Institute under US Government grants.

\section{References}

Abrahamian, H. V., \& Mickaelian, A. M. 1996, Astrophys., 39, 315

Arp, H., \& Johnson, H. L. 1955, ApJ, 122, 171 
Bade, N., Engels, D., Voges, W., et al. 1998, A\&AS, 127, 145

Bowen, I. S. 1935, ApJ, 81, 1

Casares, J., Martínez-Pais, I. G., Marsh, T. R., Charles, P. A., \& Lázaro, C. 1996, MNRAS, 278, 219

Dickinson, R. J., Prinja, R. K., Rosen, S. R., et al. 1997, MNRAS, 286, 447

Ferland, G. J. 1992, ApJ, 389, L63

Fleming, T. A., Molendi, S., Maccacaro, T., \& Wolter, A. 1995, ApJS, 99, 701

Forbes, D., \& Dawson, P. C. 1986, PASP, 98, 102

Gonçalves, A. C., Véron, P., \& Véron-Cetty, M. P. 1999, A\&A, 341,662

Hellier, C. 1996, ApJ, 471, 949

Hellier, C. 1998, PASP, 110, 420

Hellier, C., \& Robinson, E. L. 1994, ApJ, 431, L107

Hoard, D. W. 1998, Ph.D. Thesis, University of Washington

Hoard, D. W., Thorstensen, J. R., \& Szkody, P. 2000, ApJ, 537,936

Horne, K. 1999, in Annapolis Workshop on Magnetic Cataclysmic Variables, ASP Conf. Ser., 157, 349

Knigge, C., Long, K. S., Hoard, D. W., Szkody, P., \& Dhillon, V. S. 2000, ApJ, 539, L49

Lasker, B., Russel, J. N., \& Jenkner, H. 1996-99, The HST Guide Star Catalogue Version 1.1-ACT (or GSC-1.3)

Lemaître, G., Kohler, D., Lacroix, D., Meunier, J.-P., \& Vin, A. 1989, A\&A, 228, 546

McClintock, J. E., Canizares, C. R., \& Tarter, C. B. 1975, ApJ, 198,641

Mickaelian, A. M., Gonçalves, A. C., Véron-Cetty, M. P., \& Véron, P. 1999, Astrophys., 42, 1

Monet, D., Bird, A., Canzian, B., et al. 1996, USNO-A2.0, US Naval Observatory, Washington DC

Movsessian, T. H., Boulesteix, J., Gach, J.-L., et al. 2000, Baltic Astron., 9, 652

Oke, J. B. 1974, ApJS, 27, 21

Patterson, J. 1984, ApJS, 54, 443
Patterson, J. 1999, in Disk Instabilities in Close Binary Systems (Universal Academy Press), 61

Patterson, J., Kemp, K., Saad, J., et al. 1997, PASP, 109, 468

Pennington, R. L., Humphreys, R. M., Odewahn, S. C., Zumach, W., \& Thurmes, P. M. 1993, PASP, 105, 521

Roberts, D. H., Lehár, J., \& Dreher, J. W. 1987, AJ, 93, 968

Rodríguez-Gil, P., Casares, J., Martínez-Pais, I. G., Hakala, P., \& Steeghs, D. 2001, ApJ, 548, L49

Rosen, S. R., Watson, T. K., Robinson, E. R., et al. 1995, A\&A, 300, 392

Rutledge, R. E., Brunner, R. J., Prince, T. A., \& Londsdale, C. 2000, ApJS, 131, 335

Schachter, J., Filippenko, A. V., \& Kahn, S. M. 1989, ApJ, 340,1049

Schwarzenberg-Czerny, A. 1989, MNRAS, 241, 153

Shafter, A. W. 1992, ApJ, 394, 268

Shafter, A. W., Hessman, F. V., \& Zhang, E. H. 1988, ApJ, 327,248

Stone, R. P. S. 1977, ApJ, 218, 767

Taylor, C. J., Thorstensen, J. R., Patterson, J., et al. 1998, PASP, 110, 1148

Thorstensen, J. R., \& Taylor, C. J. 2000, MNRAS, 312, 629

Thorstensen, J. R., Ringwald, S. A., Wade, R. A., Schmidt, G. D., \& Norworthy, J. E. 1991, AJ, 102, 272

van Teeseling, A., \& Verbunt, F. 1994, A\&A, 292, 519

van Teeseling, A., Beuermann, K., \& Verbunt, F. 1996, A\&A, 315,467

Voges, W., Aschenbach, B., Boller, T., et al. 1999, A\&A, 349, 389

Young, P., Schneider, D. P., \& Shectman, S. A. 1981, ApJ, 244, 259

Zhang, E. R., Robinson, E. L., Ramseyer, T. F., Shetrone, M. D., \& Stiening, R. F. 1991, ApJ, 381, 534

Warner, B. 1995, Cataclysmic variable stars (Cambridge University Press), 245 